\title{
Reductive cyclization of $\delta$-hydroxy nitriles: a new synthesis of glycosylamines.
}

\author{
Jennifer E. Barbarow, Andrew D. Dorsey, and Dirk Trauner* \\ Center for New Directions in Organic Synthesis, Department of Chemistry, University of \\ California; Berkeley, CA 94720.
}

\section{Supporting Information}

General Experimental Details: Unless otherwise noted, starting materials were obtained from commercial suppliers and used without further purification. Tetrahydrofuran (THF), toluene, and dichloromethane (DCM) were dried by passing through activated alumina columns. All other solvents were analytical grade. Reactions were carried out under an atmosphere of nitrogen. Glassware, stir bars and needles were dried at $150{ }^{\circ} \mathrm{C}$ in an oven prior to use. Organic extracts were dried over $\mathrm{MgSO}_{4}$. Both reaction mixtures and chromatography fractions were analyzed on Merck silica gel $60 \mathrm{~F}_{254}$ TLC plates. Compounds were detected by treating with ceric ammonium molybdateand heating at $200{ }^{\circ} \mathrm{C}$. Flash column chromatography was carried out with ICN SiliTech 32-63 D $60 \AA$ silica gel.

Instrumentation: Melting points are uncorrected and were determined on a Büchi melting point apparatus. FT-IR spectra were obtained on $\mathrm{NaCl}$ plates with an ATI Mattson Gemini spectrometer. Proton and carbon NMR spectra $\left({ }^{1} \mathrm{H}\right.$ NMR and $\left.{ }^{13} \mathrm{C} N M R\right)$ were recorded in deuterated solvents on Bruker AM-400 or DRX-500 spectrometers and calibrated to the residual solvent peak. Multiplicities are abbreviated as follows: $\mathrm{s}=$ singlet, $\mathrm{d}=$ doublet, $\mathrm{t}=$ triplet, $\mathrm{m}=$ multiplet, app = apparent. All NMR were recorded in $\mathrm{CDCl}_{3}$. Mass spectral data were obtained from the Micro-Mass Facility operated by the College of Chemistry, University of California, Berkeley.

$(3 R, 5 \mathrm{c} R, 8 \mathrm{~b} S)$-3-Phenylhexahydro-1-oxa-3a-azacyclopentalen-4-one (1).

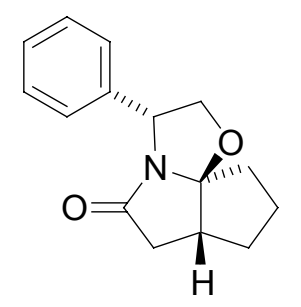

To a solution of $5.67 \mathrm{~g}$ of 2-oxocyclopentane acetic acid $(40.2 \mathrm{mmol})$ in $100 \mathrm{~mL}$ of toluene was added $5.25 \mathrm{~g}$ of $(R)-(-)-2$-phenylglycinol $(38.3 \mathrm{mmol})$. The reaction mixture was heated at reflux for $12 \mathrm{~h}$ and concentrated in vacuo. Purification by silica gel 
chromatography (3:1 hexanes:ethyl acetate) gave $8.75 \mathrm{~g}(94 \%)$ of $\mathbf{1}$ as a pale yellow oil. IR $1709 \mathrm{~cm}^{-1} .{ }^{1} \mathrm{H}$ NMR $(400 \mathrm{MHz}): \delta 1.53-1.61(\mathrm{~m}, 1 \mathrm{H}), 1.67-1.88(\mathrm{~m}, 3 \mathrm{H}), 1.92-1.97$ $(\mathrm{m}, 1 \mathrm{H}), 1.98-2.08(\mathrm{~m}, 1 \mathrm{H}), 2.42-2.57(\mathrm{~m}, 1 \mathrm{H}), 2.68-2.80(\mathrm{~m}, 1 \mathrm{H}), 2.81-2.99(\mathrm{~m}, 1 \mathrm{H})$, 3.91-4.06 (m, 1H), 4.55-4.69 (m, 1H), 5.08-5.24 (m, 1H), 7.19-7.42 (m, 5H). ${ }^{13} \mathrm{C} \mathrm{NMR}$ (100 MHz): $\delta$ 24.59, 32.40, 36.72, 40.71, 41.44, 57.90, 73.51, 110.91, 125.61, 127.49, 128.77, 139.76, 180.38. HRMS (EI) Calcd for $\mathrm{C}_{15} \mathrm{H}_{17} \mathrm{NO}_{2}\left(\mathrm{M}^{+}\right)$: 243.1259. Found: 243.1263.

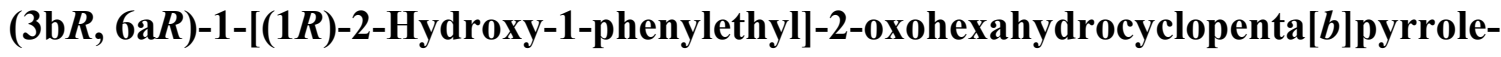
6a-carbonitrile (2).

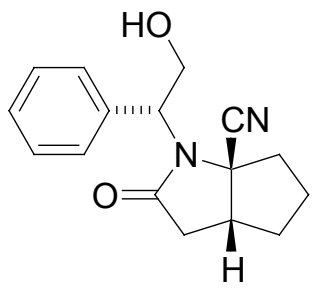

To a solution of $2.00 \mathrm{~g}$ of $1(8.23 \mathrm{mmol})$ in $100 \mathrm{~mL}$ of DCM was added $3.30 \mathrm{~mL}$ of trimethylsilyl cyanide $(24.7 \mathrm{mmol})$. The reaction mixture was cooled to $-78{ }^{\circ} \mathrm{C}$ and 16.5 $\mathrm{mL}$ of a $1.0 \mathrm{M}$ solution of titanium(IV) chloride in DCM (16.5 mmol) was added. The reaction mixture was allowed to warm to room temperature over $12 \mathrm{~h}$ and washed with 1 $\mathrm{N} \mathrm{NaOH}(3 \times 100 \mathrm{~mL})$. The organic phase was dried, filtered and concentrated in vacuo. Recrystallization from hexanes/ethyl acetate afforded $1.74 \mathrm{~g}(79 \%)$ of 2 as a white solid. m.p. $139.1-141.2^{\circ}$ C. IR 3306, 2234, $1678 \mathrm{~cm}^{-1}$. ${ }^{1} \mathrm{H}$ NMR $(500 \mathrm{MHz}): \delta 1.56-1.63$ (m, $1 \mathrm{H}), 1.74-1.88(\mathrm{~m}, 1 \mathrm{H}), 2.05-2.20(\mathrm{~m}, 2 \mathrm{H}), 2.22-2.32(\mathrm{~m}, 2 \mathrm{H}), 2.93-3.03(\mathrm{~m}, 2 \mathrm{H}), 3.06-$ $3.14(\mathrm{~m}, 1 \mathrm{H}), 3.97-4.06(\mathrm{~m}, 1 \mathrm{H}), 4.56-4.64(\mathrm{~m}, 2 \mathrm{H}), 7.33-7.52(\mathrm{~m}, 5 \mathrm{H}) .{ }^{13} \mathrm{C}$ NMR $(100$ $\mathrm{MHz}): \delta 25.34,33.73,36.70,39.15,41.08,53.73,67.90,71.22,125.67,128.16,128.93$, 135.61, 172.94, 175.62. HRMS (FAB+) Calcd for $\mathrm{C}_{16} \mathrm{H}_{19} \mathrm{~N}_{2} \mathrm{O}_{2}\left(\mathrm{M}+\mathrm{H}^{+}\right)$: 271.1447. Found: 271.1452 .

(3a $R, 6 R, 9 \mathrm{~b} R)-9-A m i n o-6-p h e n y l h e x a h y d r o-8-0 x a-5 a-a z a c y c l o p e n t a[c]$ indene-5-one (3).

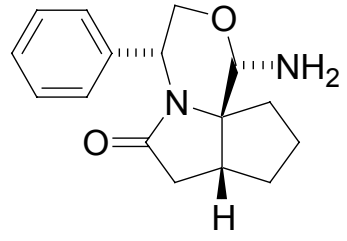

To a solution of $100 \mathrm{mg}$ of $2(0.370 \mathrm{mmol})$ in EtOH $(5.0 \mathrm{~mL})$ was added $56 \mathrm{mg}$ of sodium borohydride $(1.4 \mathrm{mmol})$. The reaction mixture was stirred at reflux for $1 \mathrm{~h}$, quenched with water and concentrated in vacuo. A 1:1 mixture of DCM/ $1 \mathrm{~N} \mathrm{NaOH}(10$ $\mathrm{mL})$ was added to the crude residue and the aqueous phase was extracted with DCM ( $3 \mathrm{x}$ $10 \mathrm{~mL}$ ). The combined organic extracts were dried, filtered and concentrated. Purification by flash column chromatography (1:9 MeOH:ethyl acetate) gave $83 \mathrm{mg}(82 \%)$ of 3 as a white solid. Recrystallization from hexanes/DCM afforded colorless, triangular, plate- 
like crystals within 3 d. For X-ray crystallographic analysis, a fragment of a colorless plate crystal of 3 having approximate dimensions of $0.35 \times 0.16 \times 0.07 \mathrm{~mm}$ was mounted on a glass fiber using Paratone $\mathrm{N}$ hydrocarbon oil. m.p. 132.5-133.3 ${ }^{\circ} \mathrm{C}$. IR 3391, 3333, $1674 \mathrm{~cm}^{-1} .{ }^{1} \mathrm{H}$ NMR $(400 \mathrm{MHz}): \delta 0.88-0.96(\mathrm{~m}, 1 \mathrm{H}), 1.32-1.50(\mathrm{~m}, 4 \mathrm{H}), 1.82-1.94(\mathrm{~m}$, $3 \mathrm{H}$ ), 2.18-2.25 (dd, $1 \mathrm{H}, J=4.8,18.0 \mathrm{~Hz}$ ), 2.49-2.59 (m, 1H), 2.70-2.79 (dd, $1 \mathrm{H}, J=10.8$, $17.6 \mathrm{~Hz}), 3.86-3.94(\mathrm{dd}, 1 \mathrm{H}, J=4.2,12.4 \mathrm{~Hz}), 4.02-4.10(\mathrm{~m}, 1 \mathrm{H}), 4.62-4.66(\mathrm{~d}, 1 \mathrm{H}, J=$ $12.4 \mathrm{~Hz}), 5.30-5.32(\mathrm{~d}, 1 \mathrm{H}, J=4.2 \mathrm{~Hz}), 7.27-7.36(\mathrm{~m}, 3 \mathrm{H}), 7.54-7.58(\mathrm{~m}, 2 \mathrm{H}) .{ }^{13} \mathrm{C}$ NMR $(100 \mathrm{MHz}): \delta 25.24,30.05,33.89,37.46,38.78,49.49,66.92,73.66,88.97,127.40$, 127.95, 128.40, 139.64, 173.91. HRMS (EI) Calcd for $\mathrm{C}_{16} \mathrm{H}_{20} \mathrm{~N}_{2} \mathrm{O}_{2}\left(\mathrm{M}^{+}\right)$: 272.1525 . Found: 272.1524 .

\section{2,3,4,6-Tetra-O-benzyl-p-D-glucopyranosylamine (7).}

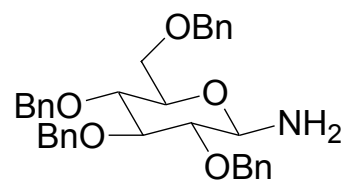

To a solution of $310 \mathrm{mg}$ of 2,3,4,6-tetra- $O$-benzyl-D-gluconitrile 6 ( $0.575 \mathrm{mmol})$ in EtOH $(18 \mathrm{~mL})$ was added $86 \mathrm{mg}$ of $\mathrm{NaBH}_{4}(2.29 \mathrm{mmol})$. The reaction mixture was stirred at room temperature for $2 \mathrm{~h}$ at which time a precipitate formed. The product was filtered, washed with cold EtOH and dried in vacuo to afford the pure $\beta$ anomer 7 as a white solid (212.4 mg, 69\%). IR 3387, $1644 \mathrm{~cm}^{-1} .{ }^{1} \mathrm{H}$ NMR (400MHz): $\delta 1.95$ (d, $\left.2 \mathrm{H}, J=8.4 \mathrm{~Hz}\right)$, $3.24(\mathrm{t}, 1 \mathrm{H}, J=12.0 \mathrm{~Hz}), 3.42-3.72(\mathrm{~m}, 6 \mathrm{H}), 4.08(\mathrm{~d}, 1 \mathrm{H}, J=8.8 \mathrm{~Hz}), 4.48-4.63(\mathrm{~m}, 3 \mathrm{H})$, 4.77-4.86 (m, 3H), 4.93-5.02 (m, 2H), 7.1-7.40 (m, 20H). Literature ${ }^{1} \mathrm{H}$ NMR $(90 \mathrm{MHz})$ : $\delta 1.94(\mathrm{~s}, 2 \mathrm{H}), 3.11-3.81(\mathrm{~m}, 6 \mathrm{H}), 4.12(\mathrm{~d}, 1 \mathrm{H}, J=9.0 \mathrm{~Hz}), 4.44-5.08(\mathrm{~m}, 8 \mathrm{H}), 6.97-7.47$ $(\mathrm{m}, 20 \mathrm{H}) .{ }^{13} \mathrm{C}$ NMR $(100 \mathrm{MHz}): \delta$ 69.22, 73.78, 75.15, 75.20, 75.81, 75.91, 78.37, 83.71, $86.03,86.48,127.81,127.86,127.93,127.95,128.04,128.14,128.19,128.26,128.33$, $128.42,128.61,128.73,128.93,138.12,138.30,138.84$. Anal Calcd for $\mathrm{C}_{34} \mathrm{H}_{37} \mathrm{NO}_{5}$ : C, 75.67; H, 6.91; N, 2.60. Found: C, 75.32; H, 7.17; N, 2.60.

\section{2-Acetamido-3,4,6-tri-O-benzyl-2-deoxy- $\beta$-D-glucopyranosylamine (9).}

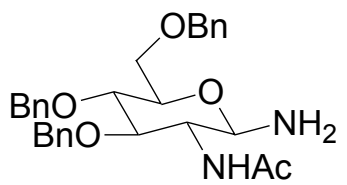

To a stirred solution of $535 \mathrm{mg}$ (1.10 mmol) of 2-acetamido-3,4,6-tri- $O$-benzyl-2-deoxy$\beta$-D-gluconitrile 8 in $10 \mathrm{~mL}$ of EtOH was added $166 \mathrm{mg}(4.40 \mathrm{mmol})$ of $\mathrm{NaBH}_{4}$. The mixture was stirred overnight, then concentrated to approximately $3 \mathrm{~mL}$. The product precipitated out of solution and was collected by filtration to afford 9 as the pure $\beta$ anomer (293 mg, 55\%). m.p. $188-189^{\circ} \mathrm{C}$. IR $3398,3281,1646 \mathrm{~cm}^{-1} .{ }^{1} \mathrm{H}$ NMR (400 MHz): $\delta 1.83(\mathrm{~s}, 3 \mathrm{H}), 2.03(\mathrm{~s}, 2 \mathrm{H}), 3.45-3.50(\mathrm{~m}, 1 \mathrm{H}), 3.53-3.78(\mathrm{~m}, 5 \mathrm{H}), 4.04(\mathrm{~d}, 1 \mathrm{H}, J=9.2$ $\mathrm{Hz}), 4.51-4.66(\mathrm{~m}, 4 \mathrm{H}) 4.79(\mathrm{~d}, 1 \mathrm{H}, J=10.8 \mathrm{~Hz}), 4.84(\mathrm{~d}, 1 \mathrm{H}, J=11.6 \mathrm{~Hz}), 5.02(\mathrm{~d}, 1 \mathrm{H}$, $J=8.8 \mathrm{~Hz}), 7.18-7.36(\mathrm{~m}, 15 \mathrm{H}) .{ }^{13} \mathrm{C}$ NMR $(100 \mathrm{MHz}): \delta 23.74,56.28,69.24,73.80$, $74.52,75.07,76.04,79.10,82.26,86.13,127.94,128.07,128.16,128.22,128.58,128.62$, 
$128.67,128.75,128.83,138.14,138.15,138.49,170.81$. HRMS (FAB) Calcd for $\mathrm{C}_{29} \mathrm{H}_{35} \mathrm{~N}_{2} \mathrm{O}_{5}\left(\mathrm{M}+\mathrm{H}^{+}\right): 491.2546$. Found: 491.2534 .

\section{2,3,4,6-Tetra-O-benzyl-D-galactopyranosylamine (11).}

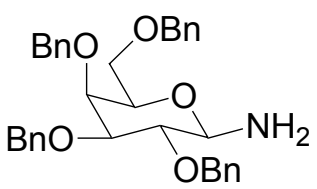

To $100 \mathrm{mg}$ of 2,3,4,6-tetra- $O$-benzyl-D-galactonitrile $10(0.186 \mathrm{mmol})$ in EtOH $(4 \mathrm{~mL})$ was added $30 \mathrm{mg}$ of $\mathrm{NaBH}_{4}(0.793 \mathrm{mmol})$. The reaction mixture was stirred for 9 hours. The solution was concentrated and the residue was dissolved in $10 \mathrm{~mL}$ of DCM. This was washed with $5 \%$ aqueous sodium bicarbonate solution $(3 \times 3 \mathrm{~mL})$. The organic layer was dried with sodium sulfate and concentrated. The resulting oil was purified by flash column chromatography (3:2 hexane: ethyl acetate) to afford a mixture of anomers, $(\alpha: \beta=1: 4,60 \mathrm{mg}, 60 \%)$. IR 3408, $3340 \mathrm{~cm}^{-1} .{ }^{1} \mathrm{H}$ NMR (500 MHz): $\delta 1.94(\mathrm{~s}, 2 \mathrm{H \alpha}, 2 \mathrm{H} \beta)$, $3.47(\mathrm{~m}, 1 \mathrm{H} \beta), 3.55-3.63(\mathrm{~m}, 2 \mathrm{H \alpha}, 4 \mathrm{H} \beta), 3.85(\mathrm{dd}, 1 \mathrm{H \alpha}, J=3.0 \mathrm{~Hz}, 9.0 \mathrm{~Hz}), 3.91(\mathrm{~d}$, $1 \mathrm{H} \beta, J=1.5 \mathrm{~Hz}$ ), 3.97 (t, $1 \mathrm{H \alpha}, J=2.5 \mathrm{~Hz}), 4.01$ (dd, $1 \mathrm{H \alpha}, J=4.0 \mathrm{~Hz}, 9.0 \mathrm{~Hz}), 4.09$ (d, $1 \mathrm{H} \beta, J=8.0 \mathrm{~Hz}$ ), 4.34 (td, $1 \mathrm{H \alpha}, J=2.0 \mathrm{~Hz}, 6.5 \mathrm{~Hz}), 4.40-4.54(\mathrm{~m}, 2 \mathrm{H \alpha}, 2 \mathrm{H} \beta), 4.59$ (d, $1 \mathrm{H \alpha}, J=11.5 \mathrm{~Hz}), 4.63(\mathrm{~d}, 1 \mathrm{H} \beta, J=11.5 \mathrm{~Hz}), 4.67(\mathrm{~d}, 1 \mathrm{H \alpha}, J=12.0 \mathrm{~Hz}), 4.72-4.79(\mathrm{~m}$, $2 \mathrm{H \alpha}, 2 \mathrm{H} \beta), 4.83-4.88(\mathrm{~m}, 1 \mathrm{H \alpha}, 1 \mathrm{H} \beta), 4.97(\mathrm{dd}, 2 \mathrm{H} \beta, J=7.5 \mathrm{~Hz}, 11.0 \mathrm{~Hz}), 5.03(\mathrm{~d}, 1 \mathrm{H \alpha}$, $J=4.5 \mathrm{~Hz}), 7.26-7.43(\mathrm{~m}, 20 \mathrm{H \alpha}, 20 \mathrm{H} \beta) .{ }^{13} \mathrm{C}$ NMR $(125 \mathrm{MHz}): \delta 69.34,73.00,73.06$, $73.45,73.50,73.72,74.04,74.29,74.41,74.72,74.86,75.53,77.08,77.43,78.17,79.15$, $79.31,80.85,83.60,86.78,127.64,127.69,127.74,127.75,127.77,127.80,127.92$, $127.98,128.05,128.16,128.38,128.40,128.41,128.45,128.48,128.51,128.52,128.53$, $128.56,128.57,128.60,137.98,138.32,138.53,138.65,138.76,138.78,138.81,138.82$. HRMS (FAB) Calcd for $\mathrm{C}_{34} \mathrm{H}_{38} \mathrm{NO}_{5}\left(\mathrm{M}+\mathrm{H}^{+}\right)$: 540.2750. Found: 540.2743 .

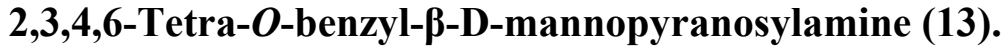

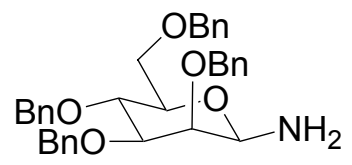

To $82 \mathrm{mg}$ of 2,3,4,6-tetra- $O$-benzyl-D-mannonitrile $12(0.153 \mathrm{mmol})$ in EtOH $(10 \mathrm{~mL})$ was added $25 \mathrm{mg}$ of $\mathrm{NaBH}_{4}(0.661 \mathrm{mmol})$. The reaction mixture was stirred for 9 hours. The mixture was concentrated and the residue was dissolved in DCM $(10 \mathrm{~mL})$. The organic phase was washed with aqueous $5 \%$ sodium bicarbonate solution $(3 \times 3 \mathrm{~mL})$. The organic layer was dried with sodium sulfate and concentrated. The oil obtained was purified by flash column chromatography (1:2 hexane: ethyl acetate) to afford the pure $\beta$ anomer 13 (57 mg, 70\%). IR $3400 \mathrm{~cm}^{-1} .{ }^{1} \mathrm{H}$ NMR (500 MHz): $\delta 2.19$ (s, 2H), 3.45-3.49 (m, 1H), 3.58-3.64 (m, 2H), $3.71(\mathrm{dd}, 1 \mathrm{H}, J=1.5 \mathrm{~Hz}, 10.0 \mathrm{~Hz}), 3.84(\mathrm{t}, 1 \mathrm{H}, J=10.0 \mathrm{~Hz})$, $3.90(\mathrm{~d}, 1 \mathrm{H}, J=2.5 \mathrm{~Hz}), 4.14$ (s, 1H), $4.50(\mathrm{~d}, 1 \mathrm{H}, J=11.0 \mathrm{~Hz}), 4.57$ (dd, $2 \mathrm{H}, J=12.5$ $\mathrm{Hz}, 18.0 \mathrm{~Hz}), 4.69$ (d, 1H, $J=11.5 \mathrm{~Hz}), 4.74$ (dd, 2H, $J=11.5 \mathrm{~Hz}, 18.5 \mathrm{~Hz}), 4.86$ (d, 1H, 
$J=10.5 \mathrm{~Hz}), 5.08(\mathrm{~d}, 1 \mathrm{H}, J=11.5 \mathrm{~Hz}), 7.12-7.15(\mathrm{~m}, 2 \mathrm{H}), 7.23-7.40(\mathrm{~m}, 18 \mathrm{H}) .{ }^{13} \mathrm{C}$ NMR (125 MHz): $\delta 69.75,72.72,73.64,75.11,75.26,75.31,76.43,83.52,84.65,127.75$, $127.81,127.83,127.85,127.94,128.16,128.22,128.24,128.49,128.51,128.52,128.67$, 138.33, 138.35, 138.37, 138.76. HRMS (FAB) Calcd for $\mathrm{C}_{34} \mathrm{H}_{37} \mathrm{NO}_{5}+\mathrm{Li}^{+}\left(\mathrm{M}+\mathrm{Li}^{+}\right)$: 546.2832. Found: 546.2843.

\section{2,3,6-tri-O-benzyl-4-O-(2,3,4,6-tetra- $O$-benzyl- $\alpha$-D-glucopyranosyl)-D-gluconitrile} (15).

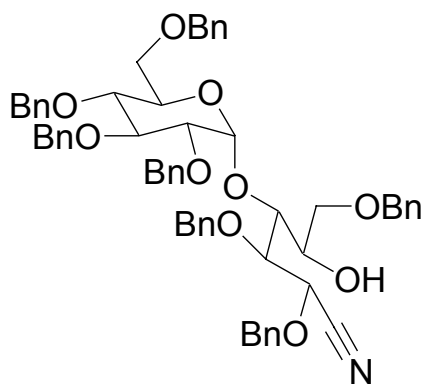

To a solution of $342 \mathrm{mg}$ of 2,3,6-tri- $O$-benzyl-4- $O$-(2,3,4,6-tetra- $O$-benzyl- $\alpha$-Dglucopyranosyl)-D-glucose oxime $14(0.346 \mathrm{mmol})$ and $192 \mathrm{mg}$ of $\mathrm{PPh}_{3}(0.730 \mathrm{mmol})$ in $\mathrm{MeCN}(3 \mathrm{~mL})$ was added $200 \mathrm{mg}$ of $\mathrm{CBr}_{4}(5.96 \mathrm{mmol})$ in $\mathrm{MeCN}(1 \mathrm{~mL})$. This was stirred for 30 minutes. Then $48 \mathrm{mg}$ of $\mathrm{PPh}_{3}(0.183 \mathrm{mmol})$ in $\mathrm{MeCN}(2 \mathrm{~mL})$ and $\mathrm{MeOH}$ $(5.2 \mathrm{~mL})$. Stirring was continued for 30 minutes and then the solution was concentrated. The oil obtained was purified by flash column chromatography (4:1 hexane: ethyl acetate) to afford 15 as a yellow oil (145 mg, 43\%). IR 3511, $2251 \mathrm{~cm}^{-1} .{ }^{1} \mathrm{H}$ NMR (500 MHz): $\delta 3.41$ (bs, $1 \mathrm{H}), 3.52$ (dd, $1 \mathrm{H}, J=6.0 \mathrm{~Hz}, 10.0 \mathrm{~Hz}), 3.58(\mathrm{~m}, 3 \mathrm{H}), 3.66(\mathrm{~d}, 1 \mathrm{H}, J=$ $10.0 \mathrm{~Hz}), 3.70(\mathrm{dd}, 1 \mathrm{H}, J=4.0 \mathrm{~Hz}, 10.5 \mathrm{~Hz}), 4.00(\mathrm{t}, 1 \mathrm{H}, J=9.5 \mathrm{~Hz}), 4.06(\mathrm{dd}, 1 \mathrm{H}, J=$ $3.0 \mathrm{~Hz}, 5.5 \mathrm{~Hz}), 4.10(\mathrm{~d}, 2 \mathrm{H}, J=11.0 \mathrm{~Hz}), 4.15(\mathrm{dd}, 2 \mathrm{H}, J=3.0 \mathrm{~Hz}, 7.5 \mathrm{~Hz}), 4.42-4.64$ $(\mathrm{m}, 7 \mathrm{H}), 4.74(\mathrm{~d}, 1 \mathrm{H}, J=11.5), 4.79-4.90(\mathrm{~m}, 5 \mathrm{H}), 4.98(\mathrm{~d}, 1 \mathrm{H}, J=11.0 \mathrm{~Hz}), 5.05(\mathrm{~d}, 1 \mathrm{H}$, $J=3.5 \mathrm{~Hz}), 7.16-7.43(\mathrm{~m}, 35 \mathrm{H}) .{ }^{13} \mathrm{C}$ NMR $(125 \mathrm{MHz}): \delta 68.60,70.97,71.02,71.24$, 71.42, 72.86, 73.47, 73.61, 74.34, 75.20, 75.40, 75.72, 78.10, 80.19, 80.42, 80.65, 82.07, $100.77,117.57,127.77,127.87,127.88,127.91,127.99,128.04,128.06,128.09,128.11$, $128.12,128.21,128.35,128.53,128.58,128.59,128.60,128.63,128.64,128.66,128.67$, 136.32, 137.48, 137.70, 137.73, 138.02, 138.03, 138.10, 138.25, 138.35, 138.90. HRMS (FAB) Calcd for $\mathrm{C}_{61} \mathrm{H}_{63} \mathrm{NO}_{10} \mathrm{Li}\left(\mathrm{M}+\mathrm{Li}^{+}\right)$: 976.4612 . Found: 976.4646 . 


\section{2,3,6-tri- $O$-benzyl-4- $O$-(2,3,4,6-tetra- $O$-benzyl- $\alpha$-D-glucopyranosyl)-D- glucopyranosyl amine (16).}

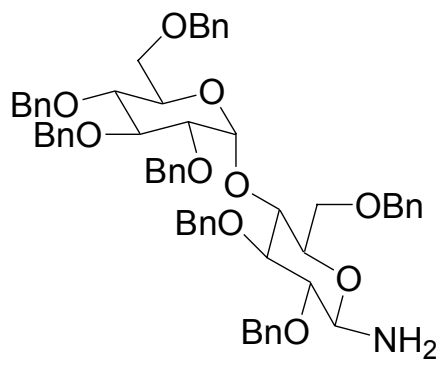

To $26 \mathrm{mg}$ of 2,3,6-tri- $O$-benzyl-4- $O$-(2,3,4,6-tetra- $O$-benzyl- $\alpha$-D-glucopyranosyl)-Dgluconitrile $15(0.026 \mathrm{mmol})$ in EtOH $(4 \mathrm{~mL})$ was added $7 \mathrm{mg}$ of $\mathrm{NaBH}_{4}(0.185 \mathrm{mmol})$. The reaction was stirred overnight and then concentrated. The residue was dissolved in DCM (10 mL) and washed with 5\% aqueous sodium bicarbonate solution (2 x $2 \mathrm{~mL})$. The organic layer was dried with sodium sulfate and concentrated. The resulting oil was purified by flash column chromatography (1:1 hexane: ethyl acetate) to afford $\mathbf{1 6}$ as a mixture of anomers $(\alpha: \beta=1: 4,13 \mathrm{mg}, 50 \%)$. IR $3417 \mathrm{~cm}^{-1} .{ }^{1} \mathrm{H}$ NMR $(500 \mathrm{MHz}): \delta 1.64$ (bs, $2 \mathrm{H \alpha}), 1.98$ (bs, $2 \mathrm{H} \beta), 3.27(\mathrm{t}, 1 \mathrm{H} \beta, J=8.5 \mathrm{~Hz}), 3.39-5.02(\mathrm{~m}, 27 \mathrm{H \alpha}, 26 \mathrm{H} \beta), 5.58(\mathrm{~d}$, $1 \mathrm{H} \alpha, J=5.0 \mathrm{~Hz}), 5.66(\mathrm{~d}, 1 \mathrm{H} \beta, J=5.0 \mathrm{~Hz}), 7.10-7.40(\mathrm{~m}, 35 \mathrm{H} \alpha, 35 \mathrm{H} \beta) .{ }^{13} \mathrm{C} \mathrm{NMR}(125$ $\mathrm{MHz}): \delta 66.04,68.42,71.27,73.52,73.56,73.69,75.18,75.36,75.73,76.98,77.48$, $79.51,82.23,126.74,127.69,127.70,127.84,127.89,127.91,127.98,128.06,128.22$, $128.44,128.47,128.49,128.51,128.54,128.55,138.15,138.40,139.04$. HRMS (FAB) Calcd for $\mathrm{C}_{61} \mathrm{H}_{66} \mathrm{NO}_{10}\left(\mathrm{M}+\mathrm{H}^{+}\right)$: 972.4687. Found: 972.4713 .

\section{2,3,5-Tri-O-benzyl-D-ribonitrile (21).}

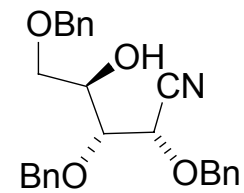

To a solution of $1.33 \mathrm{~g}$ of 2,3,5-tri- $O$-benzyl-D-ribose oxime $(3.19 \mathrm{mmol})$, and $1.67 \mathrm{~g}$ of $\mathrm{PPh}_{3}(6.38 \mathrm{mmol})$ in $\mathrm{MeCN}(25.5 \mathrm{~mL})$ was added $2.64 \mathrm{~g} \mathrm{CBr}_{4}(7.97 \mathrm{mmol})$ in $\mathrm{MeCN}$ $(10 \mathrm{~mL})$. This was stirred of 20 minutes. Then $0.42 \mathrm{~g}$ of $\mathrm{PPh}_{3}(1.59 \mathrm{mmol})$ in $\mathrm{MeCN}$ $(12.5 \mathrm{~mL})$ and $\mathrm{MeOH}$ in $(35.8 \mathrm{~mL})$ was added. The solution was stirred for an additional 15 minutes and concentrated. The oil obtained was purified by flash column chromatography (3:1 hexane: ethyl acetate) to afford 21 as an oil $(820 \mathrm{mg}(62 \%)$. IR 3557, 3467, 3089, 3067, 3033, 2915, 2871, 2253, $\mathrm{cm}^{-1} .{ }^{1} \mathrm{H}$ NMR (500 MHz): $\delta 2.48$ (s, $1 \mathrm{H}), 3.55$ (dd, $1 \mathrm{H}, J=4.5 \mathrm{~Hz}, 9.5 \mathrm{~Hz}), 3.59$ (dd, $1 \mathrm{H}, J=3.0 \mathrm{~Hz}, 9.5 \mathrm{~Hz}), 3.83-3.90$ (m, $2 \mathrm{H}), 4.46(\mathrm{dd}, 2 \mathrm{H}, J=12.0 \mathrm{~Hz}, 28 \mathrm{~Hz}), 4.57(\mathrm{t}, 2 \mathrm{H}, J=11.5 \mathrm{~Hz}), 4.70(\mathrm{~d}, 1 \mathrm{H}, J=3.0$ $\mathrm{Hz}), 4.91(\mathrm{dd}, 2 \mathrm{H}, J=8.0 \mathrm{~Hz}, 11.0 \mathrm{~Hz}), 7.26-7.41(\mathrm{~m}, 15 \mathrm{H}) .{ }^{13} \mathrm{C}$ NMR $(125 \mathrm{MHz}): \delta$ 69.89, 70.12, 71.21, 73.04, 73.61, 74.64, 78.95, 116.54, 128.12, 128.16, 128.18, 128.40, 128.49, 128.59, 128.66, 128.71, 128.86, 136.01, 137.59, 137.72. HRMS (FAB) Calcd for $\mathrm{C}_{26} \mathrm{H}_{27} \mathrm{NO}_{4} \mathrm{Li}\left(\mathrm{M}+\mathrm{Li}^{+}\right)$: 424.2100. Found: 424.2097. 
$\beta$-N-9-fluorenylmethyloxycarbonyl- (N-2,3,4,6-Tetra-O-benzyl- $\beta$-D-glucopyranosyl) aspartic acid allyl ester (27).

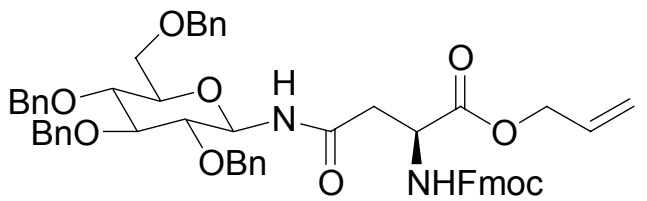

To $110 \mathrm{mg}$ of 2,3,4,6-tetra- $O$-benzyl- $\beta$-D-glucopyranosylamine $7(0.20 \mathrm{mmol})$ and $97 \mathrm{mg}$ of $N$ - $\alpha$-Fmoc-L-aspartic acid $\alpha$-allyl ester $26(0.24 \mathrm{mmol})$ in $10 \mathrm{~mL}$ of THF at $-15^{\circ} \mathrm{C}$ was added $28 \mathrm{mg}$ of HOBt $(0.20 \mathrm{mmol})$ and $51 \mathrm{mg}$ of DCC $(0.24 \mathrm{mmol})$ in $5 \mathrm{~mL}$ of THF. The reaction mixture was stirred at $-15{ }^{\circ} \mathrm{C}$ for 1 hour, then was allowed to warm to room temperature. Stirring was continued at room temperature overnight. The reaction was filtered, DCM $(50 \mathrm{~mL})$ was added to the filtrate, and the resulting solution was washed successively with $5 \%$ aqueous citric acid $(3 \times 8 \mathrm{~mL}), 5 \%$ aqueous sodium bicarbonate ( 3 x $8 \mathrm{~mL}$ ), and water ( $3 \times 8 \mathrm{~mL})$. The organic layer was dried and concentrated. The solid obtained was purified by flash column chromatography (5:1 hexane: ethyl acetate) to afford 27 (89.2 mg, 49\%). m.p. 199-200 ${ }^{\circ} \mathrm{C}$. IR 3301, 1739, 1699, 1665, $1073 \mathrm{~cm}^{-1} .{ }^{1} \mathrm{H}$ NMR (500 MHz): $\delta 2.43(\mathrm{dd}, 1 \mathrm{H} J=4.0 \mathrm{~Hz}, 16.5 \mathrm{~Hz}), 2.72(\mathrm{dd}, 1 \mathrm{H}, J=4.0 \mathrm{~Hz}, 16.5$ $\mathrm{Hz}), 3.21-3.25(\mathrm{~m}, 1 \mathrm{H}), 3.39$ (d, 1H, $J=8.0 \mathrm{~Hz}$ ), 3.56-3.68 (m, 4H), $4.13(\mathrm{t}, 1 \mathrm{H}, J=7.3$ $\mathrm{Hz}), 4.20(\mathrm{dd}, 1 \mathrm{H}, \mathrm{J}=7.5 \mathrm{~Hz}, 10.5 \mathrm{~Hz}), 4.29-4.62(\mathrm{~m}, 8 \mathrm{H}), 4.70-4.74(\mathrm{~m}, 2 \mathrm{H}), 4.82(\mathrm{~s}$, $2 \mathrm{H}), 4.93(\mathrm{t}, 1 \mathrm{H}, J=9.0 \mathrm{~Hz}), 5.15(\mathrm{~d}, 1 \mathrm{H}, J=10.5 \mathrm{~Hz}), 5.24(\mathrm{~d}, 1 \mathrm{H}, J=17.0 \mathrm{~Hz}), 5.35$ (d, $1 \mathrm{H}, J=8.5 \mathrm{~Hz}), 5.78-5.86(\mathrm{~m}, 1 \mathrm{H}), 5.96(\mathrm{~d}, 1 \mathrm{H}, J=8.5 \mathrm{~Hz}), 7.02-7.04(\mathrm{~m}, 2 \mathrm{H}), 7.10-$ $7.31(\mathrm{~m}, 22 \mathrm{H}), 7.52(\mathrm{~d}, 2 \mathrm{H}, J=7.5 \mathrm{~Hz}), 7.67(\mathrm{~d}, 2 \mathrm{H}, J=7.5 \mathrm{~Hz}) .{ }^{13} \mathrm{C}$ NMR $(125 \mathrm{MHz}): \delta$ $37.79,47.25,50.61,66.52,67.46,68.15,73.73,74.64,75.16,75.99,76.49,77.64,78.85$, $79.27,86.30,118.84,120.13,125.40,125.46,127.27,127.85,127.86,127.96,127.98$, $127.99,128.01,128.09,128.24,128.55,128.59,128.67,128.95,129.19,131.82,137.82$, 138.01, 138.13, 138.43, 141.41, 141.43, 143.98, 144.04, 156.36, 170.36, 170.76. HRMS (FAB) Calcd for $\mathrm{C}_{56} \mathrm{H}_{56} \mathrm{~N}_{2} \mathrm{O}_{10}\left(\mathrm{M}+\mathrm{H}^{+}\right)$: 917.4013. Found: 917.4011 .

\section{$\beta$ - $N$-9-fluorenylmethyloxycarbonyl-( $N$-2-acetamido-3,4,6-tri- $O$-benzyl- $\beta$-D- glucopyranosyl) aspartic acid allyl ester (28).}

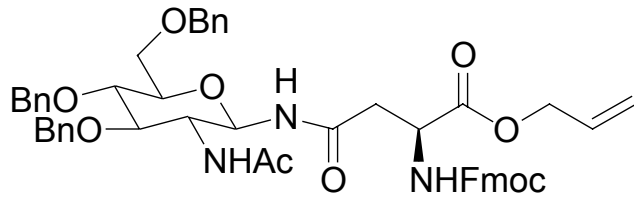

$80 \mathrm{mg}$ of $N$ - $\alpha$-Fmoc-L-aspartic acid $\alpha$-allyl ester $26(0.204 \mathrm{mmol})$ and $62 \mathrm{mg}$ of 2 isobutoxy-1-isobutoxycarbonyl-1,2-dihydroquinoline (IIDQ) $(0.204 \quad \mathrm{mmol}) \quad$ were dissolved in DCM $(15 \mathrm{~mL})$ and the mixture was stirred for 2 minutes. To this mixture was added $50 \mathrm{mg}$ of 2-acetamido-3,4,6-tri-O-benzyl-2-deoxy- $\beta$-D-glucopyranosylamine $(0.102 \mathrm{mmol})$ and stirring was continued for $1 \mathrm{~h}$. The reaction was monitored by TLC (1:9 hexane: ethyl acetate). After $1 \mathrm{~h}$ the reaction mixture was concentrated and the solid was redissolved in DCM $(40 \mathrm{~mL})$ and this solution was extracted with $5 \%$ aqueous 
sodium bicarbonate solution $(7 \times 8 \mathrm{~mL})$ and water $(4 \times 8 \mathrm{~mL})$. The organic layer was dried and concentrated. The product was crystallized from acetone to remove unreacted starting material and afford the $\beta$ linked 28 (45 mg, 50\%). m.p. 233-235 ${ }^{\circ} \mathrm{C} .{ }^{1} \mathrm{H}$ NMR $(500 \mathrm{MHz}): \delta 1.68(\mathrm{~s}, 3 \mathrm{H}), 2.69$ (dd, $1 \mathrm{H}, J=4.0 \mathrm{~Hz}, 16.5 \mathrm{~Hz}), 2.88(\mathrm{dd}, 1 \mathrm{H}, J=4.5 \mathrm{~Hz}$, $16.5 \mathrm{~Hz}), 3.41(\mathrm{dd}, 1 \mathrm{H}, J=8.5 \mathrm{~Hz}, 10.5 \mathrm{~Hz}), 3.47(\mathrm{~d}, 1 \mathrm{H}, J=9.5 \mathrm{~Hz}), 3.71-3.87(\mathrm{~m}, 4 \mathrm{H})$, $4.22(\mathrm{t}, 1 \mathrm{H}, J=7.5 \mathrm{~Hz}), 4.28$ (dd, $1 \mathrm{H}, J=7.5 \mathrm{~Hz}, 10.5 \mathrm{~Hz}), 4.41$ (dd, $1 \mathrm{H}, J=7.0 \mathrm{~Hz}$, $10.5 \mathrm{~Hz}), 4.47(\mathrm{~d}, 1 \mathrm{H}, J=12.5 \mathrm{~Hz}), 4.56-4.68(\mathrm{~m}, 8 \mathrm{H}), 4.74(\mathrm{dd}, 1 \mathrm{H}, J=8.5 \mathrm{~Hz}, 9.0 \mathrm{~Hz})$, $4.79(\mathrm{~d}, 1 \mathrm{H}, J=11 \mathrm{~Hz}), 4.86(\mathrm{~d}, 1 \mathrm{H}, J=12.5 \mathrm{~Hz}), 5.17(\mathrm{dd}, 1 \mathrm{H}, J=1.5 \mathrm{~Hz}, 10.5 \mathrm{~Hz})$, $5.26(\mathrm{dd}, 1 \mathrm{H}, J=1.5 \mathrm{~Hz}, 18 \mathrm{~Hz}), 5.81-5.89(\mathrm{~m}, 1 \mathrm{H}), 6.06(\mathrm{~d}, 1 \mathrm{H}, J=9.5 \mathrm{~Hz}), 7.17-7.40$ $(\mathrm{m}, 19 \mathrm{H}), 7.59$ (d, $2 \mathrm{H}, J=7.2 \mathrm{~Hz}), 7.74(\mathrm{~d}, 2 \mathrm{H}, J=7.6 \mathrm{~Hz}) .{ }^{13} \mathrm{C}$ NMR $(125 \mathrm{MHz}): \delta$ 23.27, 37.67, 47.31, 53.65, 66.32, 67.50, 68.18, 73.88, 74.45, 75.33, 76.72, 77.43, 78.57, $80.29,80.95,118.51,120.15,127.30,127.88,127.89,128.03,128.26,128.31,128.36$, 128.64, 128.76, 128.86, 129.26, 129.28, 131.86, 137.86, 138.09, 141.44, 141.48, 171.02, 171.20, 172.39, 172.67. HRMS (FAB) Calcd for $\mathrm{C}_{51} \mathrm{H}_{53} \mathrm{~N}_{3} \mathrm{O}_{10}+\mathrm{Li}^{+}\left(\mathrm{M}+\mathrm{Li}^{+}\right): 874.3891$. Found: 874.3914. 


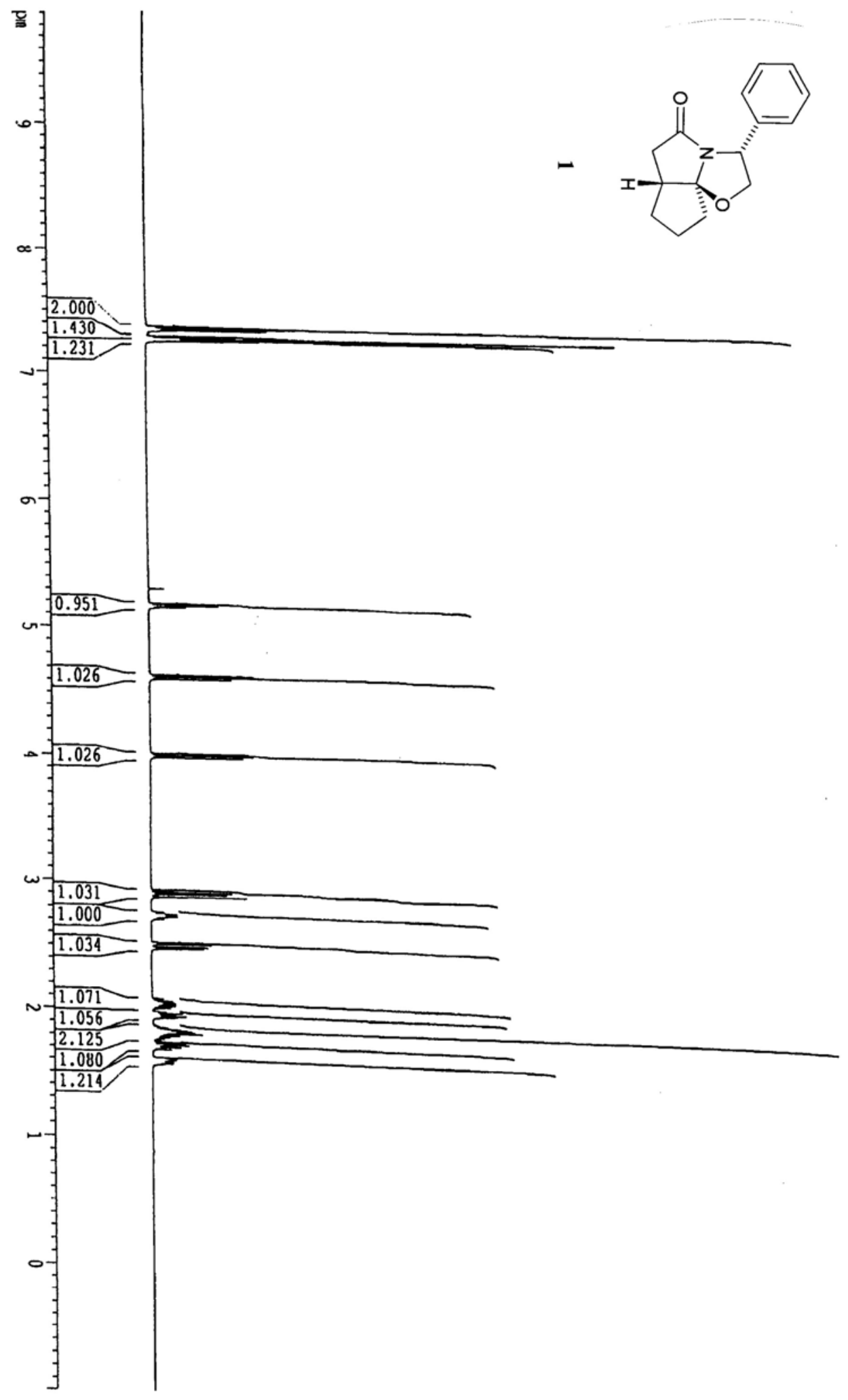




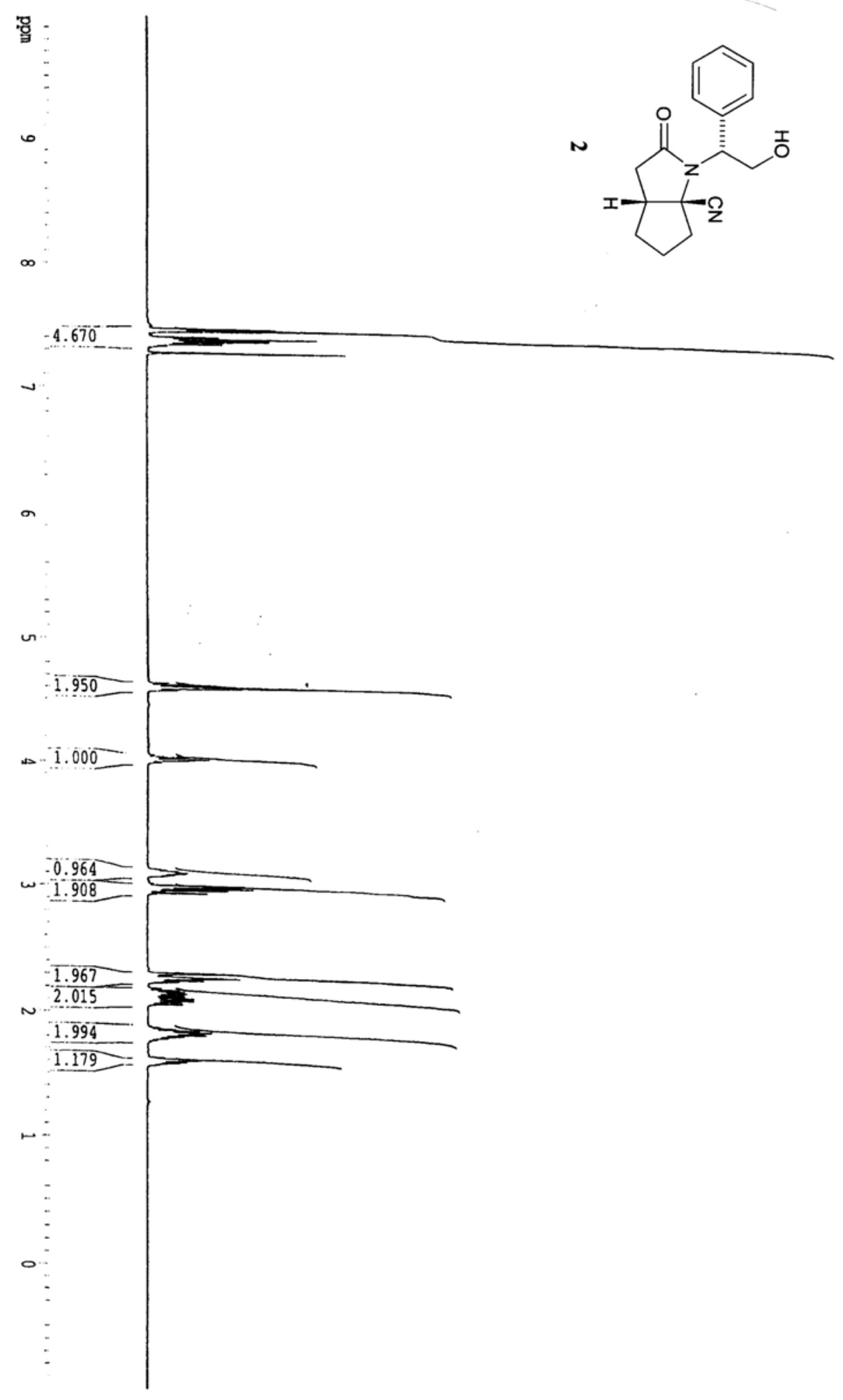




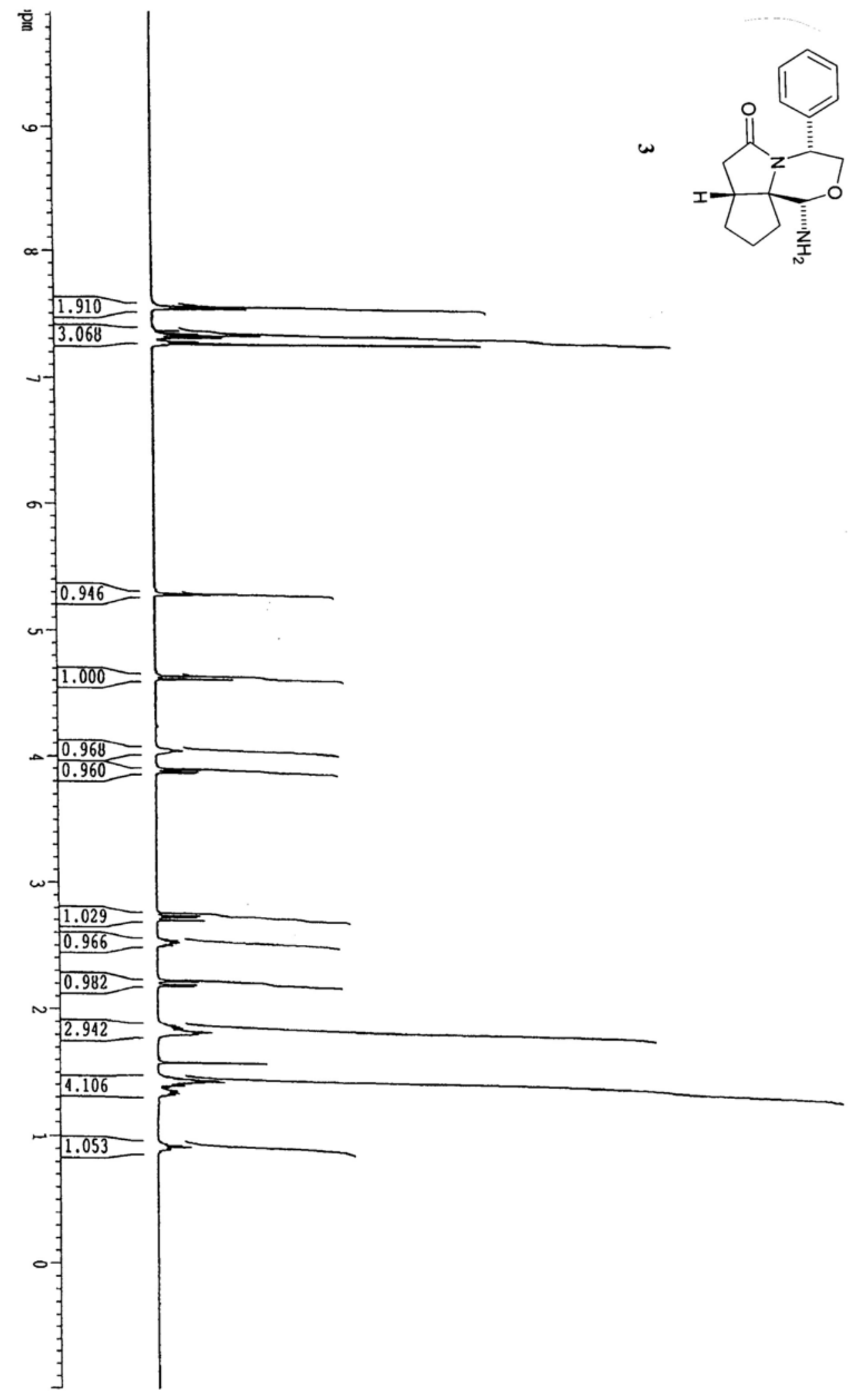




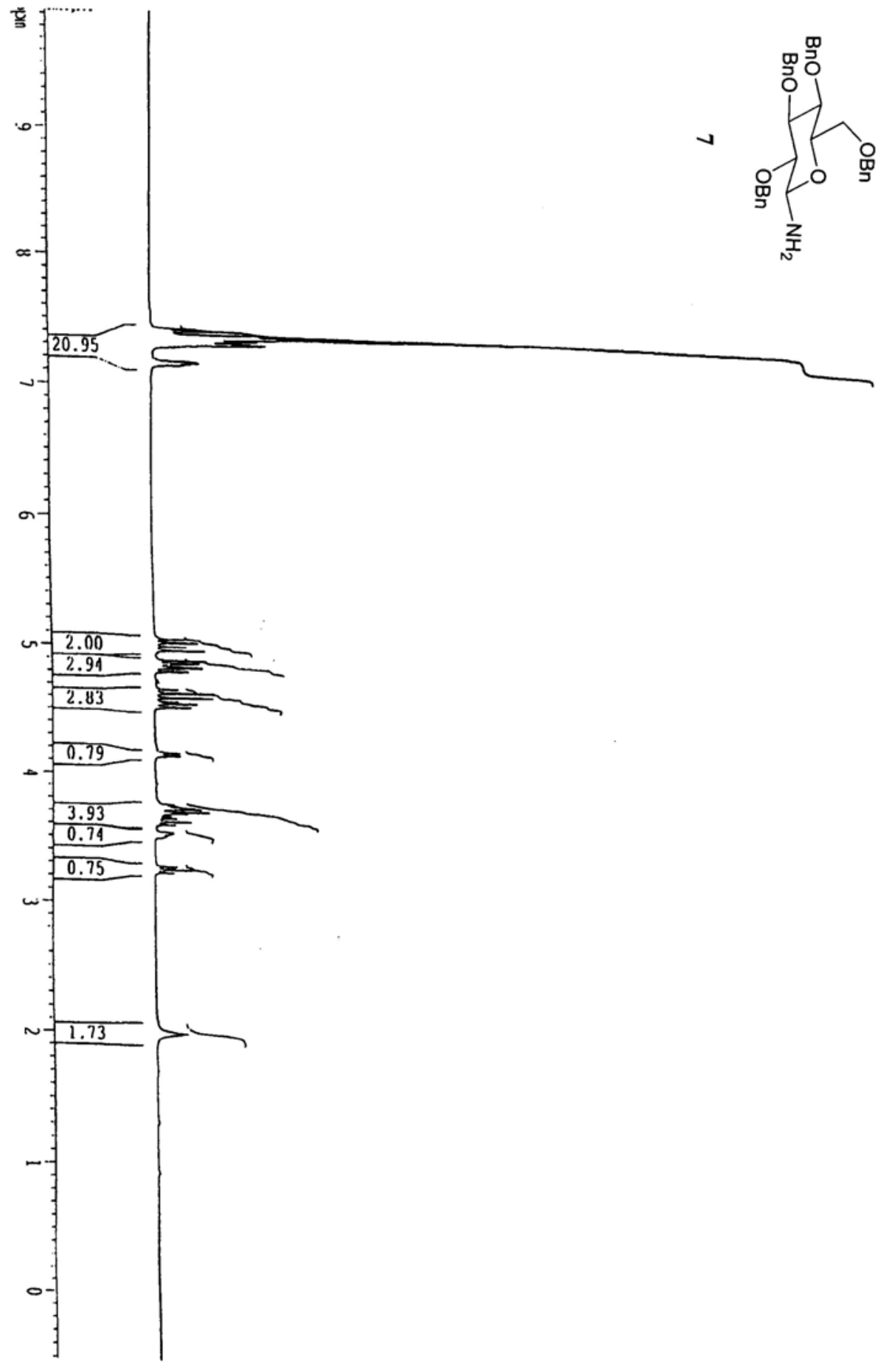




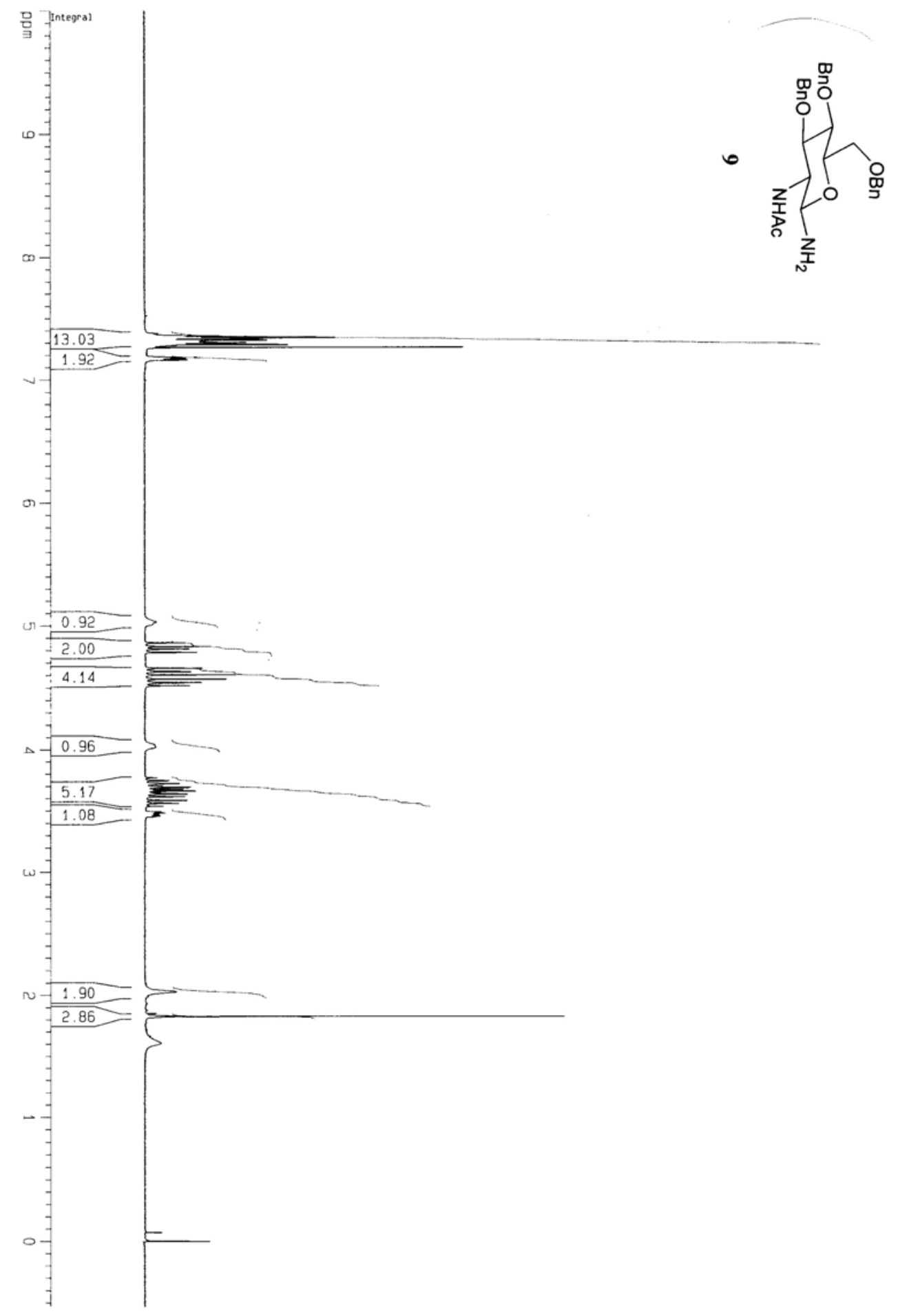




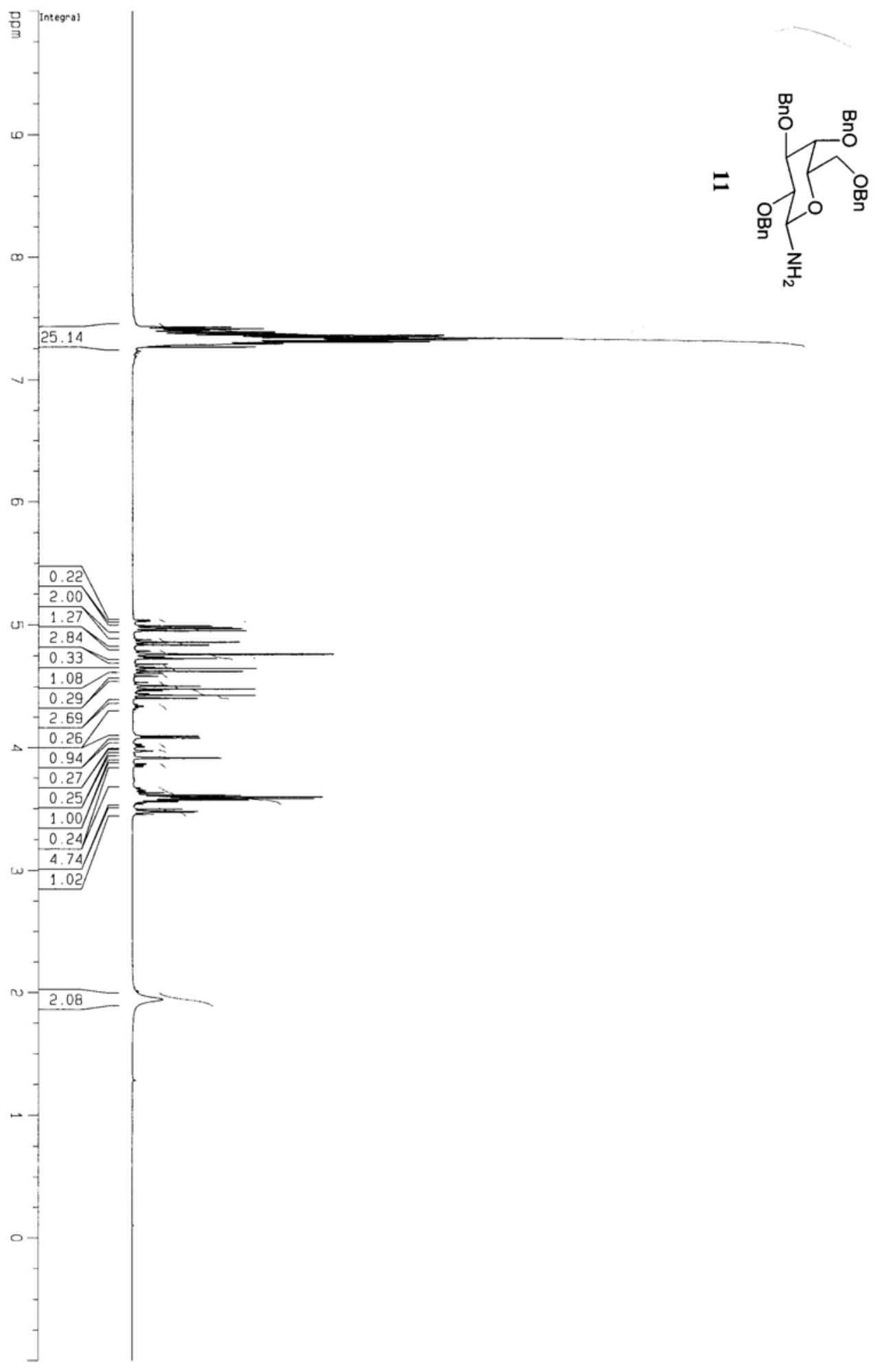




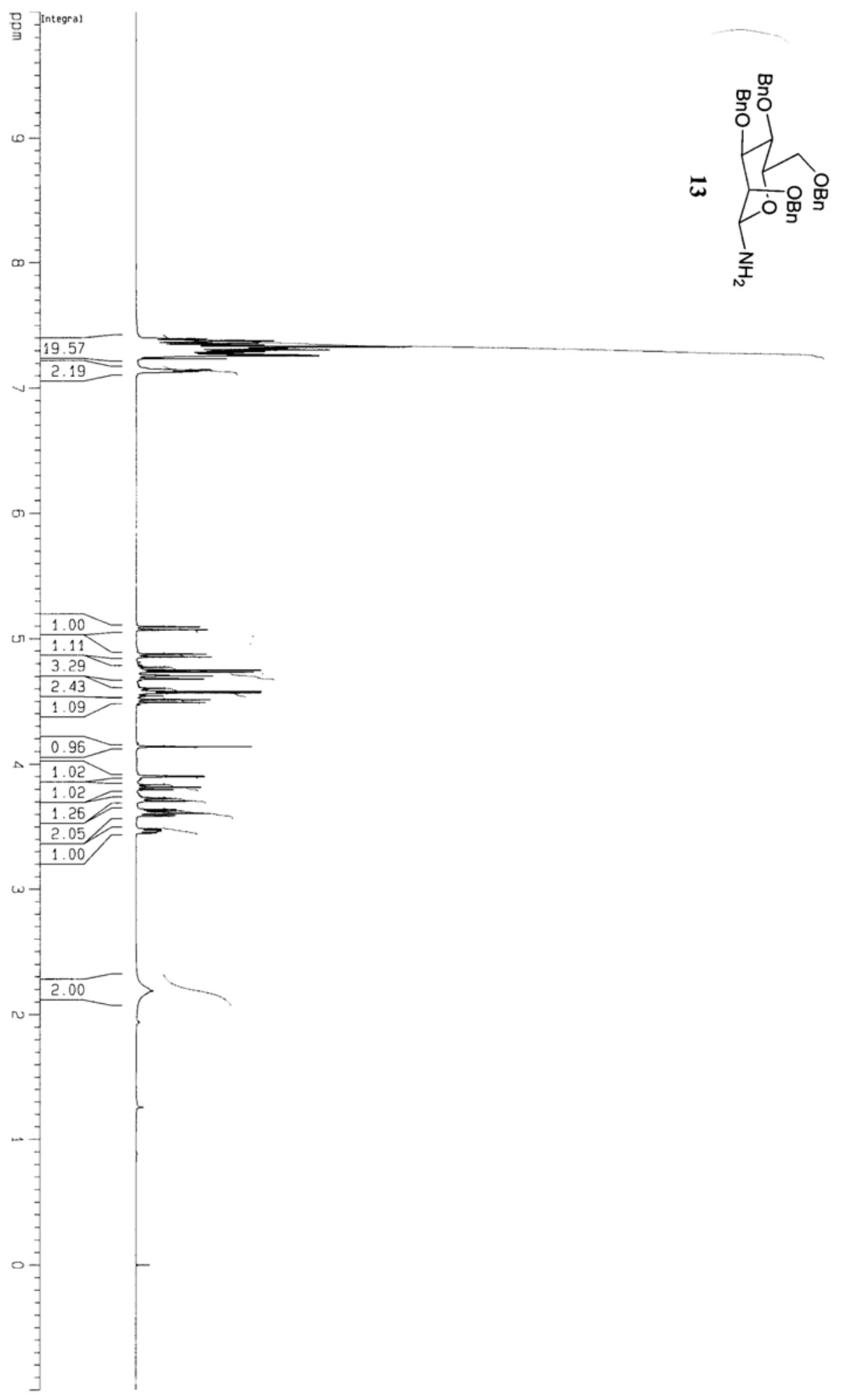




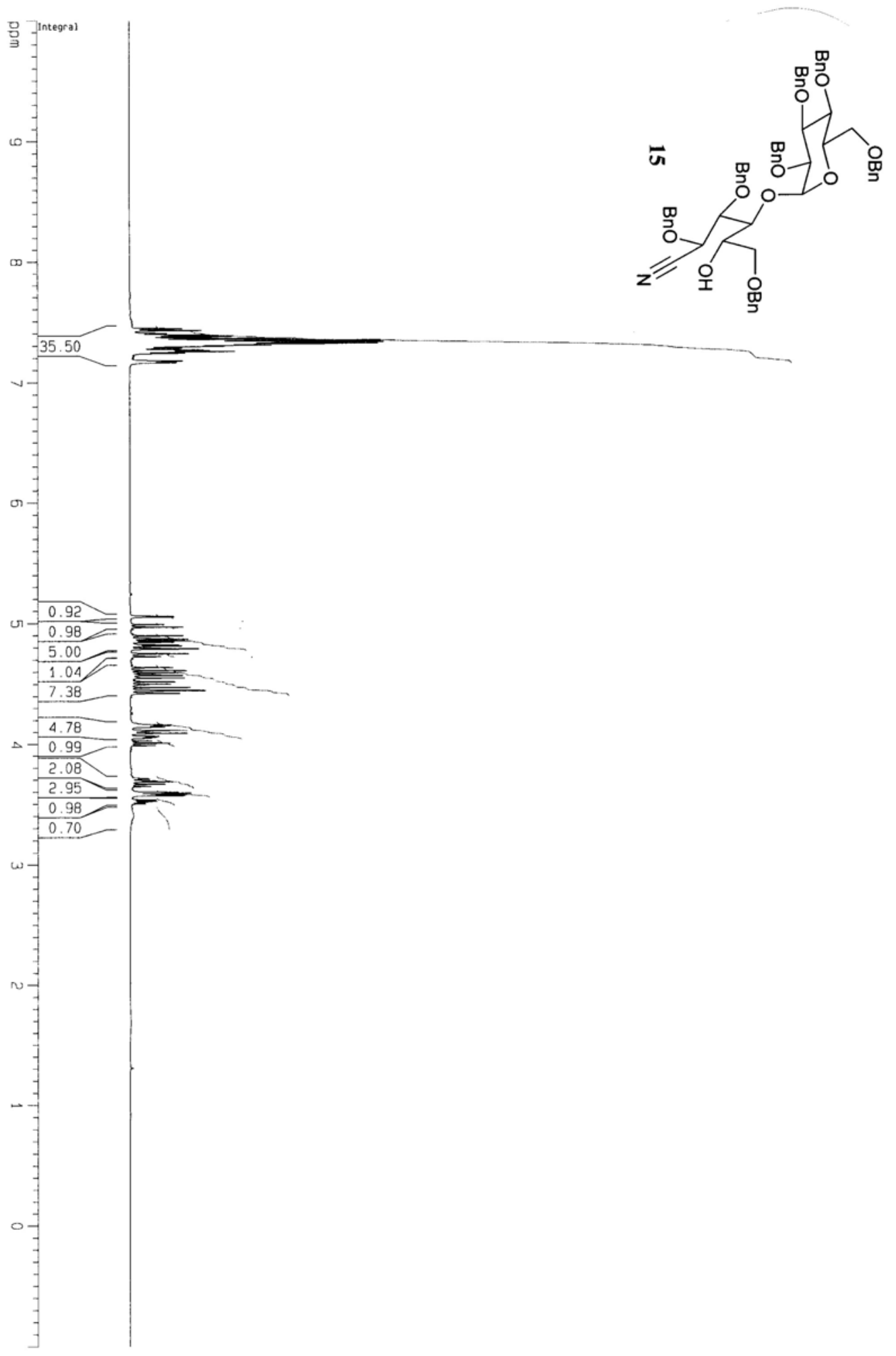




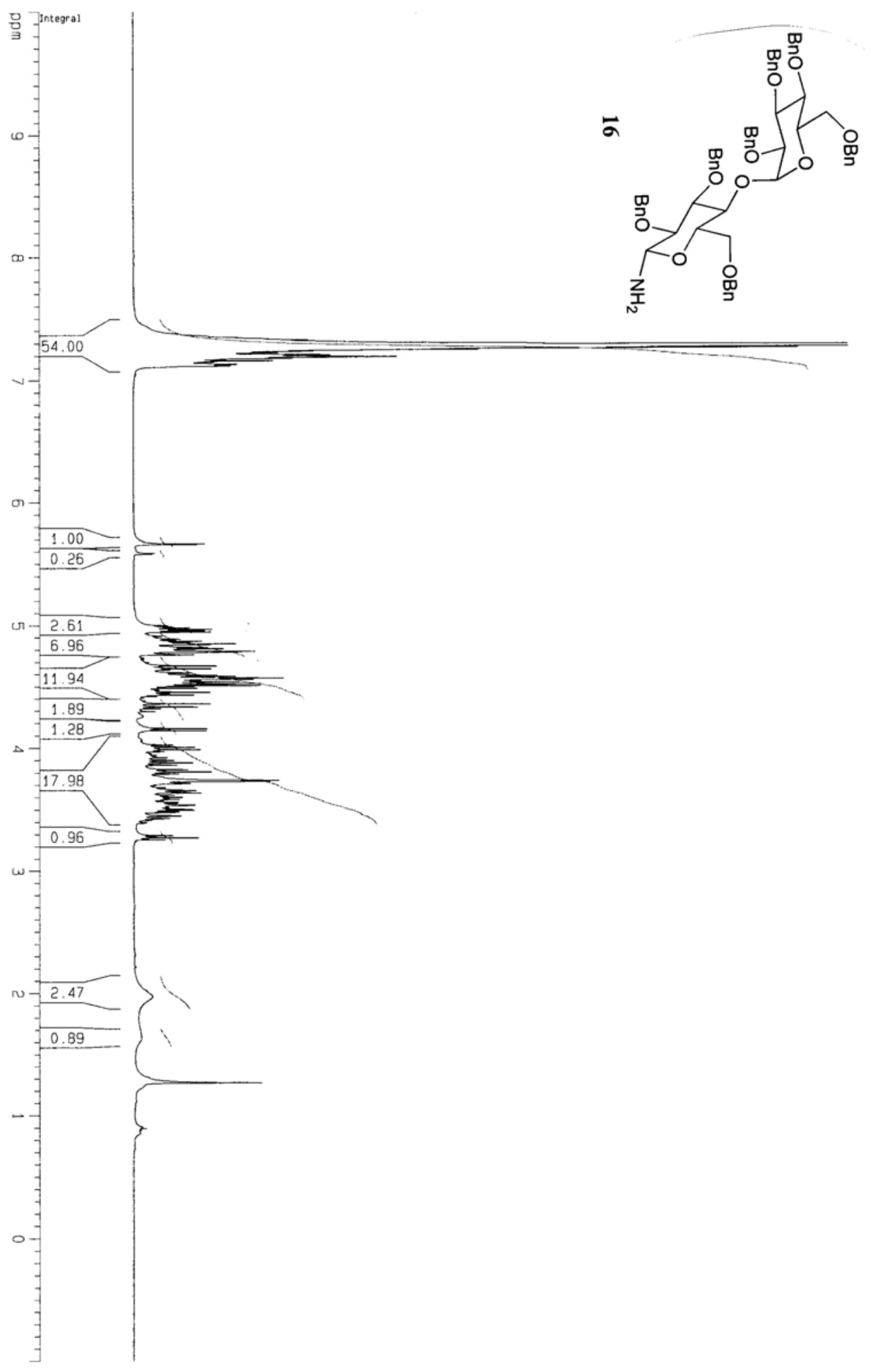




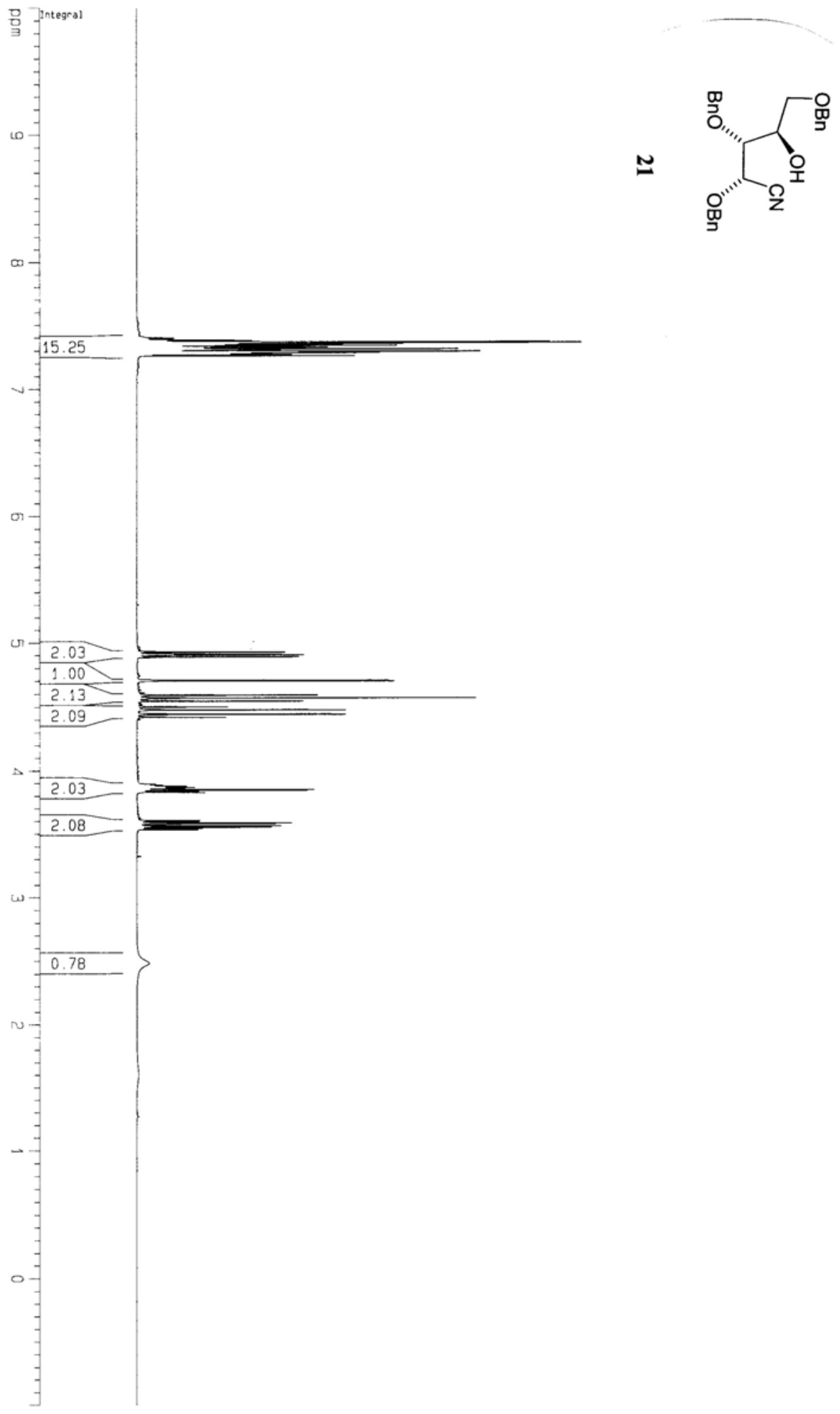




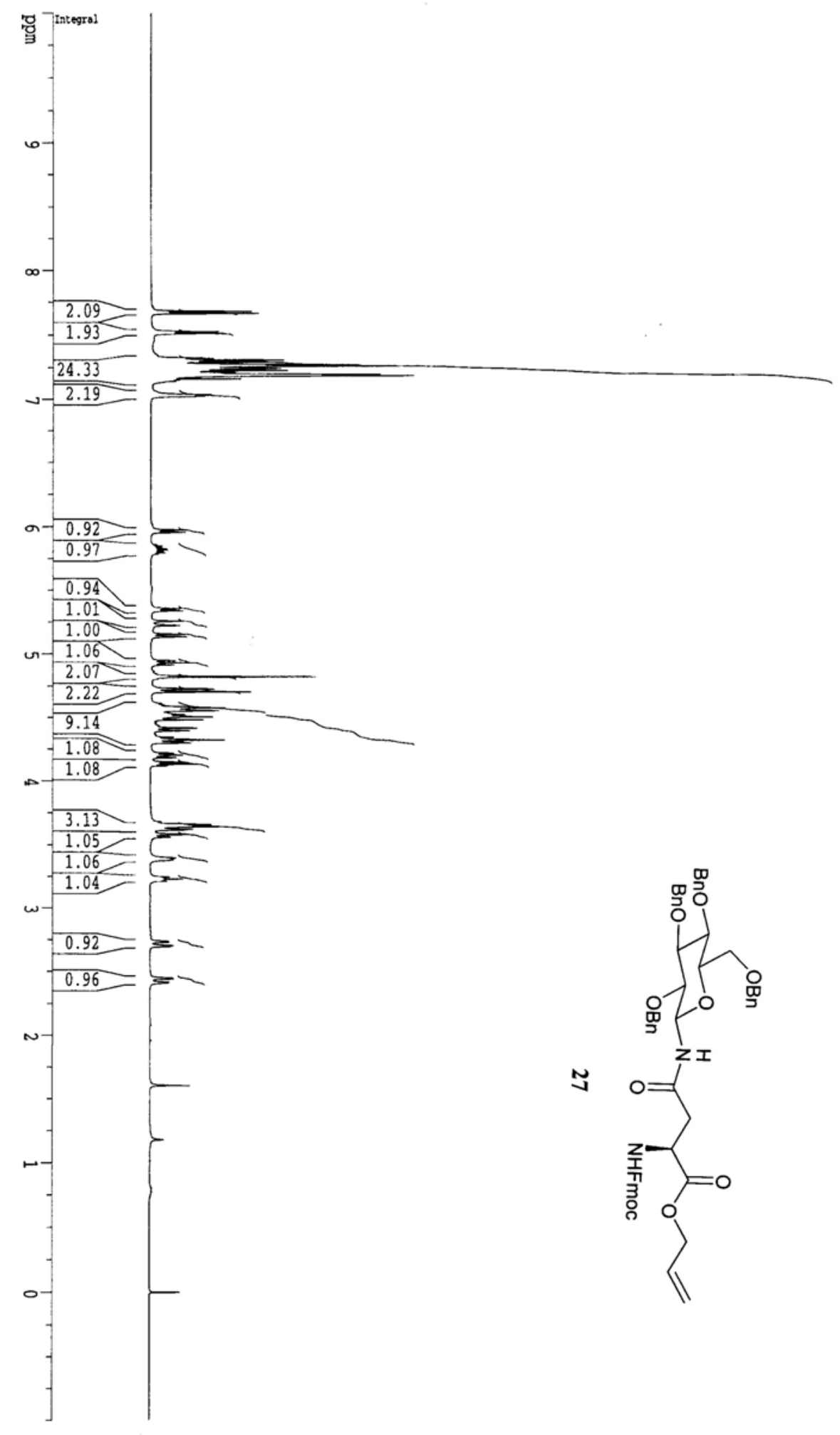




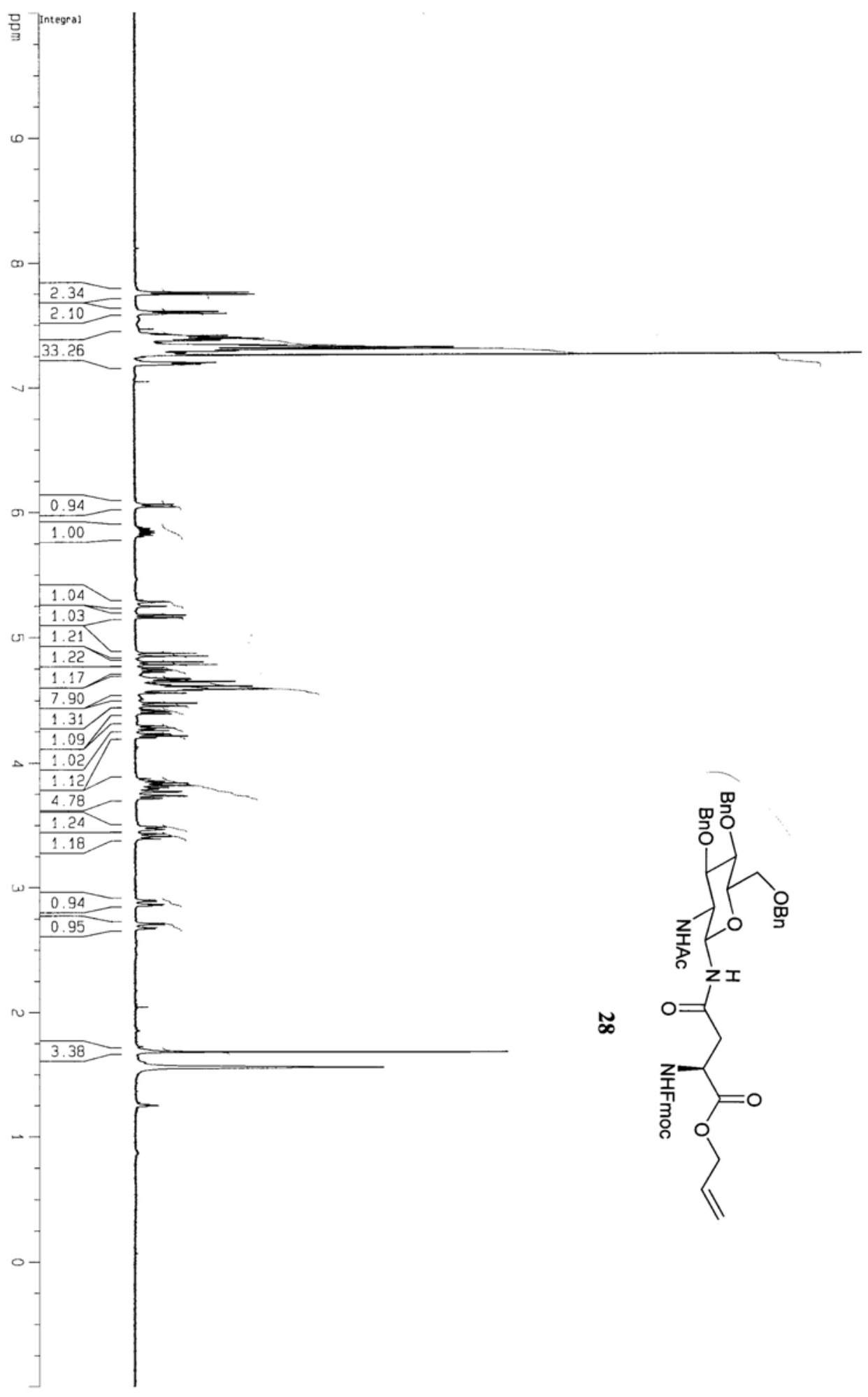

